\title{
Use of Failure Mode and Effect Analysis to Reduce Admission of Asymptomatic COVID-19 Patients to the Adult Emergency Department: An Institutional Experience
}

This article was published in the following Dove Press journal:

Risk Management and Healthcare Policy

\author{
Berhanetsehay Teklewold (iD) \\ Dagmawi Anteneh ${ }^{2}$ \\ Dawit Kebede ${ }^{2}$ \\ Wendmagegn Gezahegn ${ }^{3}$ \\ 'Department of Surgery, St. Paul's \\ Hospital Millennium Medical College, \\ Addis Ababa, Ethiopia; ${ }^{2}$ Clinical \\ Governance and Quality Improvement \\ Directorate, St. Paul's Hospital \\ Millennium Medical College, Addis Ababa, \\ Ethiopia; ${ }^{3}$ Department of Paediatrics and \\ Child Health, St. Paul's Hospital \\ Millennium Medical College, Addis Ababa, \\ Ethiopia
}

\begin{abstract}
Background: Failure mode and effect analysis is an important tool to identify failures in a system with its possible cause, effect, and set actions to be implemented proactively before the occurrence of problems. This study tries to identify common failure modes with its possible causes and effect to the health service and to plot actions to be implemented to reduce COVID-19 transmission to clients, staff, and subsequent service compromise from asymptomatic COVID-19 patients visiting the adult emergency department of SPHMMC (non-COVID-19 setup).
\end{abstract}

Method and Study Design: A multidisciplinary team, representing different divisions of the adult emergency department at St. Paul's Hospital Millennium Medical College (SPHMMC), was chosen. This team was trained on failure mode and effect analysis and basics of COVID-19, to identify possible causes of failures and their potential effects, to calculate a risk priority number (RPN) for each failure, and plan changes in practice.

Results: A total of 22 failure modes and 89 associated causes and effects were identified. Many of these failure modes (12 out of 22) were found in all steps of patient flow and were associated with either due to lack of or failure to apply standard and transmission-based precautions. This suggests the presence of common targets for improvement, particularly in enhancing the safety of staff and clients. As a result of this FMEA, 23 general improvement actions were proposed.

Conclusion: FMEA can be used as a useful tool for anticipating potential failures in the process and proposing improvement actions that could help in reducing secondary transmissions during the pandemic.

Keywords: COVID-19, FMEA, standard precaution

\section{Introduction}

On February 11, 2020, the World Health Organization (WHO) named the disease caused by severe acute respiratory syndrome coronavirus (SARS-CoV-2) "COVID19 ", and as the number of cases and deaths increased in several countries, its pandemic status was declared on March $11,2020 .{ }^{1}$ Ethiopia also reported it's first COVID-19 infected patient 2 days after the global pandemic declaration. ${ }^{2}$

COVID-19 patients might be asymptomatic or symptomatic and when symptomatic they might present with conditions of mild-to-severe respiratory difficulties which might even necessitate admission to the intensive care unit. ${ }^{3}$ A systematic
Correspondence: Berhanetsehay Teklewold Department of Surgery, St. Paul's Hospital Millennium Medical College, P.O. Box I27I, Addis Ababa, Ethiopia

Tel +251944308583

Email Berhanetsehay.teklewold@sphmmc. edu.et 
review by Yanes-Lane et $\mathrm{al}^{4}$ has shown a higher proportion of asymptomatic infection in COVID-19 patients with significantly higher transmission potential.

During the initial phase of the pandemic, there can be a rapid growth of cases related to asymptomatic patients and this is epidemiologically important as they represent a silent source of spread in various settings like emergency rooms in a hospital set-up. ${ }^{5}$

When a pandemic of this extent and a huge spectrum of symptoms challenges the health system, the risk of misdiagnosing a patient might increase and the safety of patients might be compromised. It is therefore essential to anticipate the types of diagnostic challenges that can occur and build reliable systems that can limit failures from occurring to support the safety of both patients and clinicians. Improving the flow of patients in and out of healthcare settings can help to improve the safety and quality of care. $^{6}$

Several improvement tools are being used in health system delivery to improve the safety of processes, one of which is Failure Mode and Effect Analysis. ${ }^{7}$

This tool tries to determine all possible failures to measure the seriousness of the outcome and the frequency of reasons that bring about the failure setting up the precedent actions that help in improving the flow and design of processes. ${ }^{8,9}$ Several studies have shown the impact of the tool in quality improvement initiatives in a different hospital setting and also various processes in care delivery. ${ }^{10,11}$

The basic idea behind FMEA lies on the assumption that risks are linked not only to the odds of failure happening but also to the severity of its outcome and the feasibility of detecting and stopping before it unfolds. ${ }^{12,13}$

During the COVID-19 pandemic, using the FMEA tool might be of use to anticipate potential failures that could occur during admission and transfer of patients in emergency rooms at a non-COVID-19 set-up. This study tries to identify possible common failures that could cause admission of COVID-19 patients to a non-COVID-19 emergency set-up and by doing so to limit possible transmission to patients and staff in the set-up and to generate recommendations for patients and staff safety.

\section{Methodology}

\section{Study Setting}

The project was done in the adult emergency department of SPHMMC, Addis Ababa, Ethiopia (a tertiary teaching hospital). SPHMMC is one of the largest hospitals in Ethiopia, located in the capital. Currently, it is providing both COVID-19 and non-COVID-19 related healthcare services from all walks of life. The COVID-19 treatment center, Isolation ward, and sampling and testing for COVID-19 were among the COVID related service areas at SPHMMC. The non-COVID-19 related healthcare services which were being provided before the pandemic continued with set-up modification. Among these, an adult emergency is one of the service areas, which provides services to medical and surgical emergencies for patients above 14 years of age. It provides services to patients ranging between $25-30$ per day. ${ }^{14}$ The project period was from March to May 2020.

\section{Team Selection}

The team consists of five physicians (two from the emergency department with a specialty in emergency and critical care, two from quality control, and one from IPCInfection prevention and control), four senior nurses, one nurse assistant, one cleaner, and one security guard working in the adult emergency department.

\section{Study Population}

The study population consisted of patients and attendants visiting the adult emergency department, physicians, nurses, nurse assistants (runners), cleaners, and security guards working in the adult emergency department.

\section{Study Design and Method}

The cross-sectional study was conducted on patients, attendants, and staff working in and around the adult emergency department.

The team studies the complex process of screening for COVID-19, triaging, management, and disposal of patients in the emergency setup.

The study consists of four stages which include process mapping that identifies the steps in accessing emergency service flow diagram (Figure 1); the second stage is listing potential indicators of failures that can predispose to COVID-19 transmission at each step. In the third stage, it explores also possible reasons for the occurrence of failure and its effect followed by prioritizing failure modes based on the specific risk priority numbers (RPN). ${ }^{12}$ All team members will give an approximate score on the probability of occurrence of failure, its severity, and the probability of its detectability before it could take place. All scores will be added to get maximum RPN 


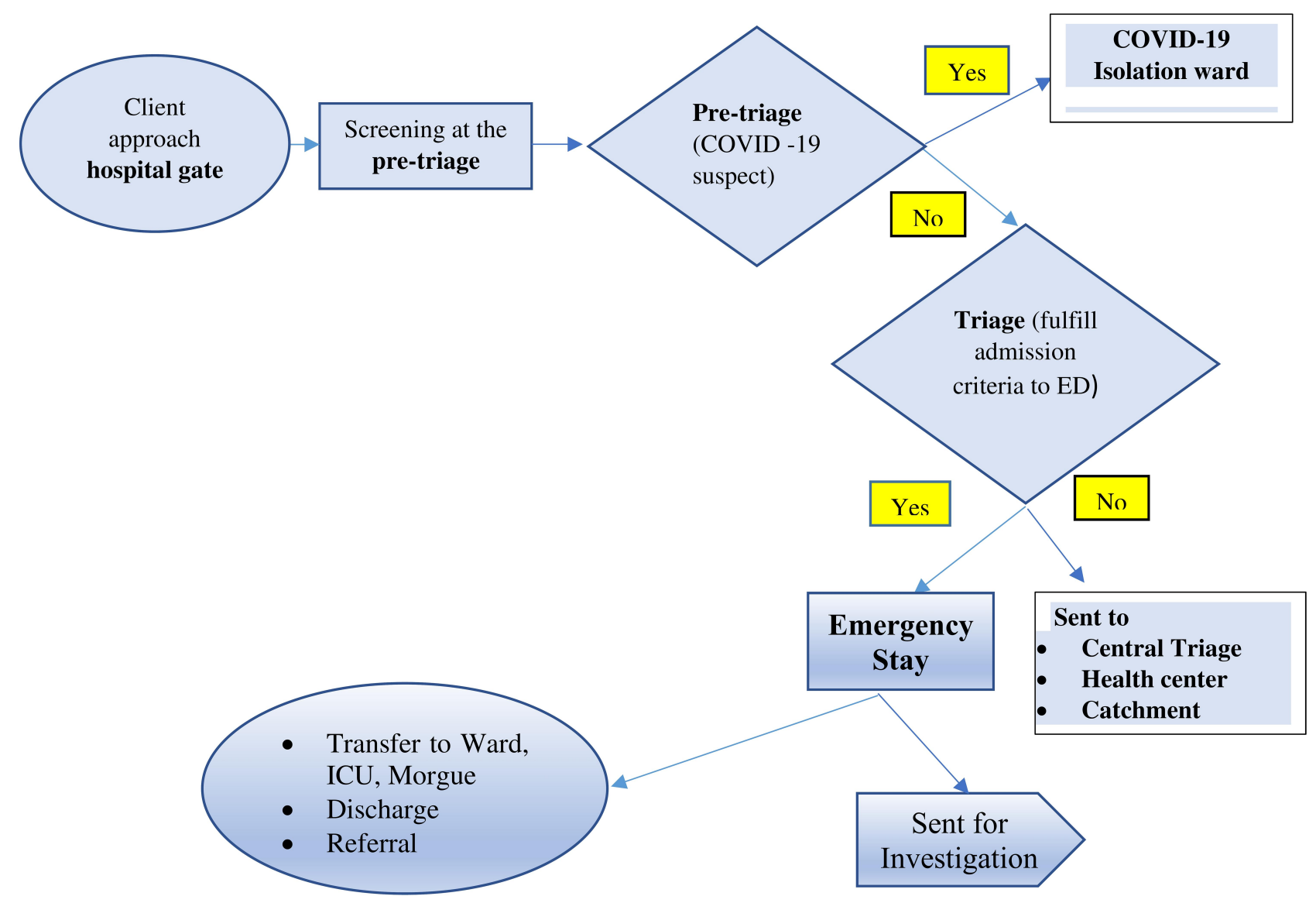

Figure I Process map showing patient flow in the adult emergency department of SPHMMC during the COVID-19 pandemic.

and, at the final stage, different recommendations on mechanisms of preventing such failures will be identified. Details of scores attached to each of the processes are described in Table 1.

Ethical approval was given by the institutional review board of St Paul hospital Millennium medical college (reference number: PM23/247) and informed consent was taken from participant's during observation and the aim and outcome of the study was explained accordingly. The study was conducted in accordance with the Declaration of Helsinki.

\section{Results}

\section{Process Mapping}

The process map for patient flow in the adult emergency department of SPHMMC is as follows in Figure 1.

Based on the process map identified in Figure 1, 22 failure modes and 89 possible causes and effects attributed were distinguished with 81 possible recommendations suggested to limit the occurrence of failures on each stage of patient movement from the entry gate of the hospital until the patient is transferred/referred or discharged from the emergency department. The RPN identified runs in the interval of 30-336. On the entry gate of the hospital, screening is done to reduce the chance of letting in possible COVID-19 suspect patients, which is done by asking the history for risk factors and symptoms and assessing signs.

At the hospital gate entry, five failure modes that could make COVID-19 transmission easy were identified with 21 associated causes, and 18 measures were plotted to tackle the failures. RPN of these failure modes ranges between 30-112. That patient and attendant do not apply standard precautions was the failure mode identified that scored the highest RPN at this step (Table 2).

At pre-triage, patients coming to the adult emergency department further evaluated whether they are further COVID-19 suspect or not. If they fulfil COVID-19 suspect criteria, they will be transferred to the isolation ward but if they fail to fulfil the criteria they will be triaged and evaluated in the emergency department. Six high-risk failures were detected in total with 27 attributed causes and 20 


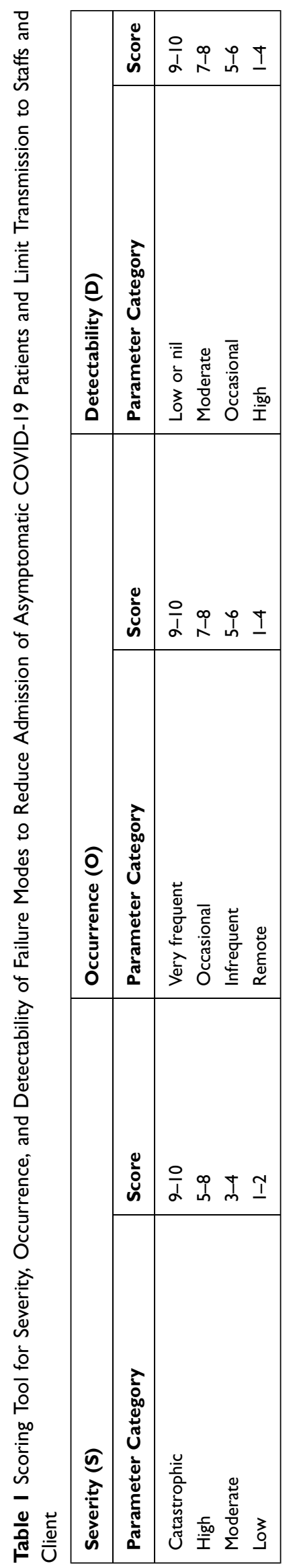

possible recommendations to be applied proactively. The high-risk failure at this point is pre-triage does not detect the case (COVID 19) (RPN-192) (Table 3).

In the triage room of the adult emergency department, six failure modes with 20 possible causes and 19 measures to be taken were suggested. The failure mode identified with maximum RPN (336) in this area is a low index of suspicion, and the minimum RPN (42) was patient bypasses the triage area (Table 4 ).

The adult emergency room has separate zones for patient management based on severity score as green, yellow, orange, red, and black. During this time multiple failures might happen that can predispose to or increase COVID-19 transmission. A total of five failure modes with 21 possible causes were identified and 25 actions to be implemented were plotted. RPN of this failure modes ranges between 60-280. A low index of suspicion was the failure mode that has scored the highest RPN (280) (Table 5).

\section{Discussion}

We performed FMEAs in the adult emergency department to go through with the potential loopholes associated with delivering service to patients who visit our emergency department. We found that processes of emergency service differ hugely according to the difference in patient clinical condition and were highly exposed to many failure modes; however, most vulnerabilities identified were common to all processes in the emergency department. These play a great role in devising common improvement actions that can be implemented easily. Using FMEA proactively to simulate potential failures during the COVID-19 pandemic has been shown by other simulation studies too that has used FMEA risk analysis to identify potential failures that could occur in transferring COVID-19 patients. ${ }^{15}$ A study by Levy et $\mathrm{al}^{16}$ has also shown how the FMEA tool has helped them in identifying hazards and implement mitigation strategies in their effort during the establishment of a field hospital during the COVID-19 pandemic.

The most critical process that could increase COVID19 transmission at the entry gate of the hospital is that clients do not apply standard precautions when they came to the hospital $(\mathrm{RPN}=112)$. This most high-risk failure mode occurs most of the time and is believed to result from a lack of knowledge about precautions (standard and transmission-based), lack of compliance, lack of monitoring mechanism for compliance of precautions, and shortage or absence of precaution facility. 


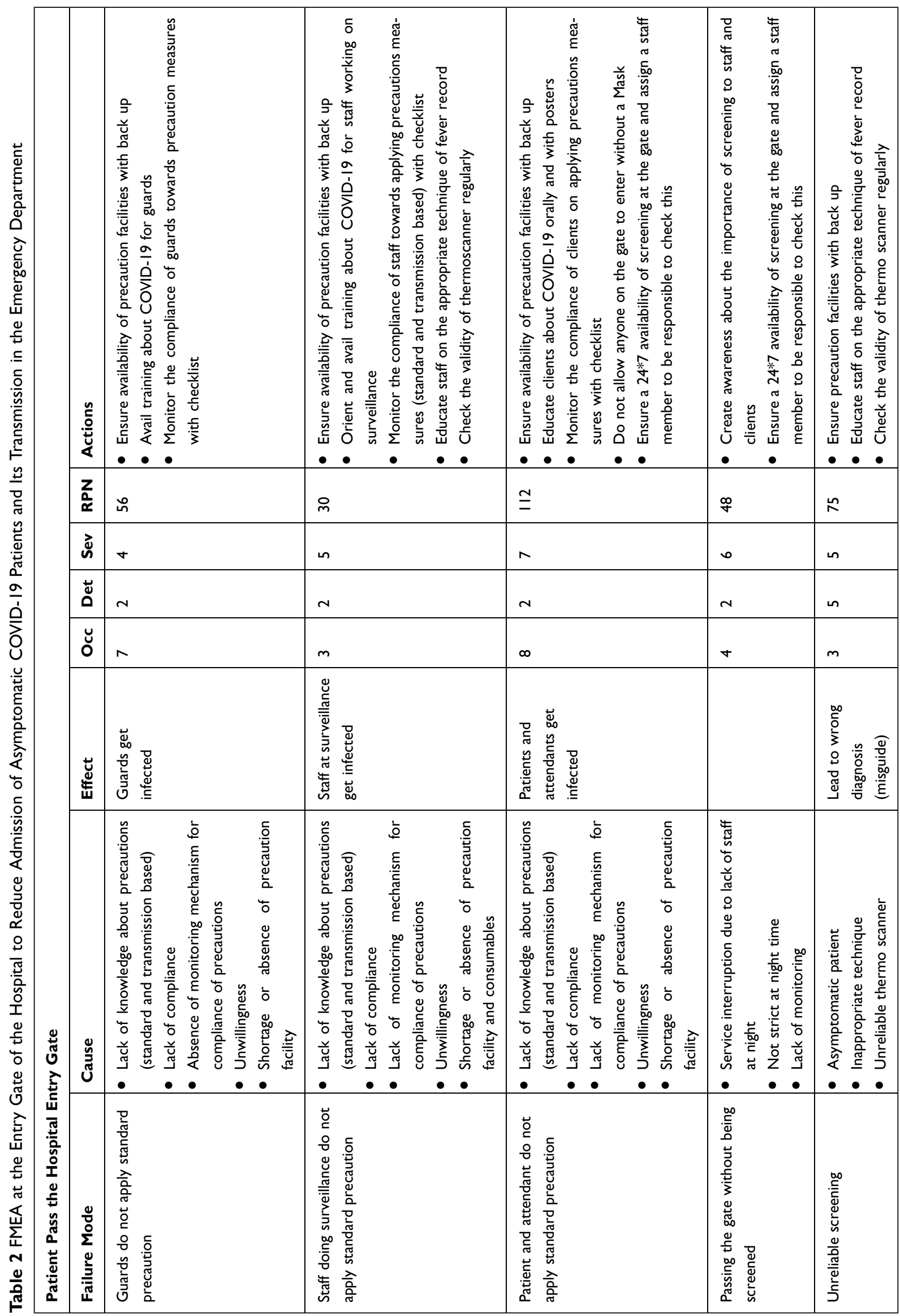




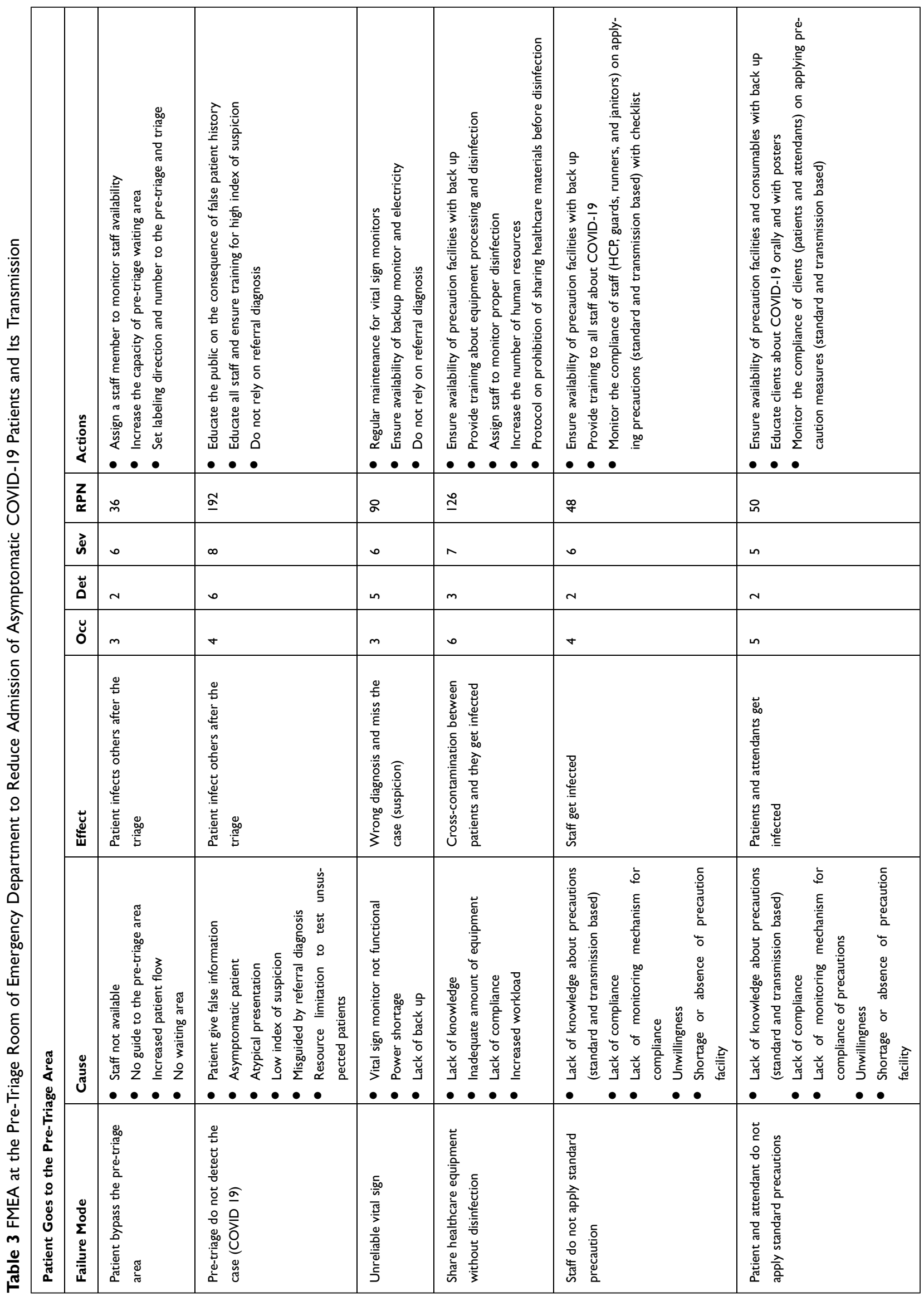




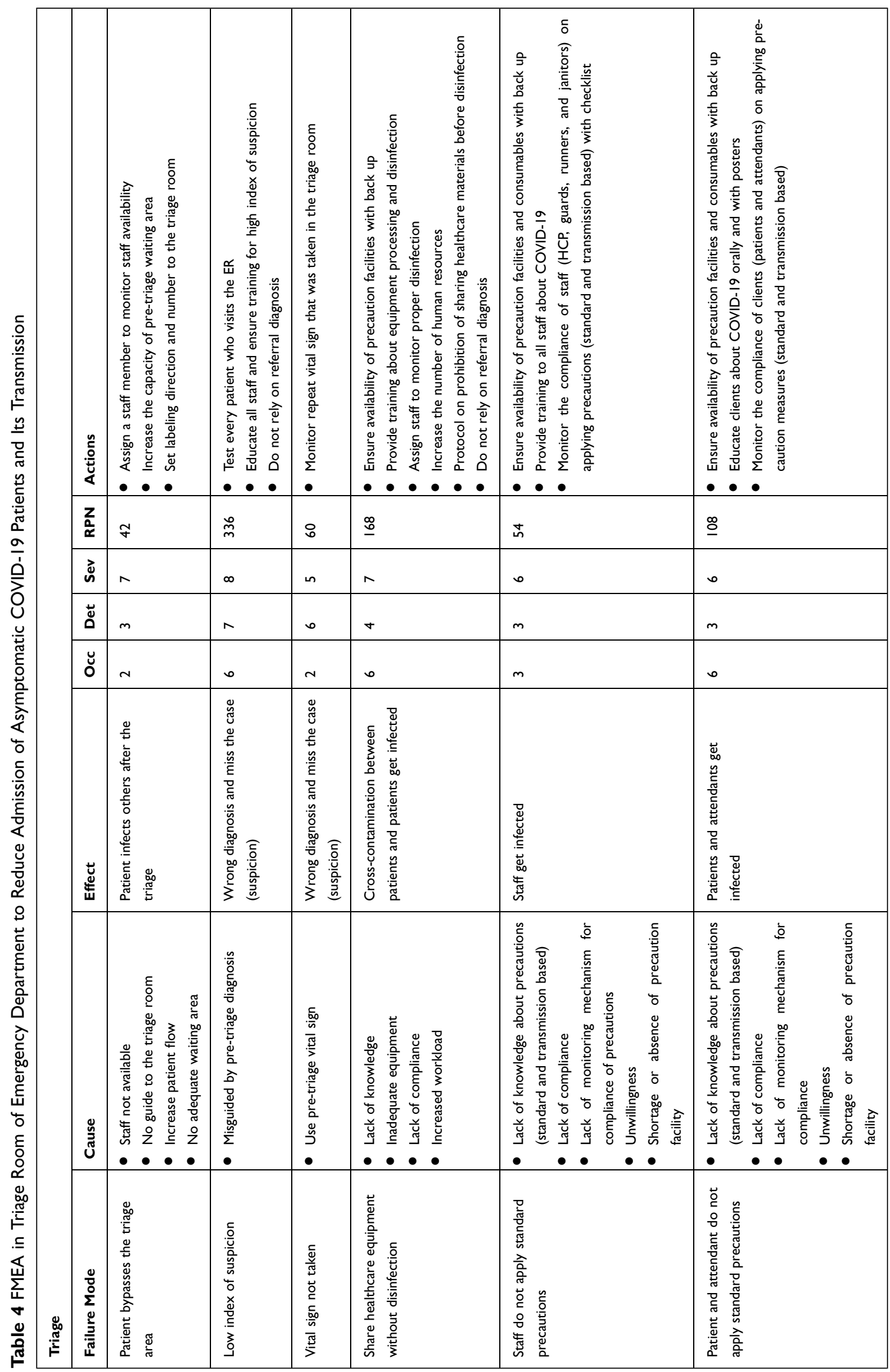




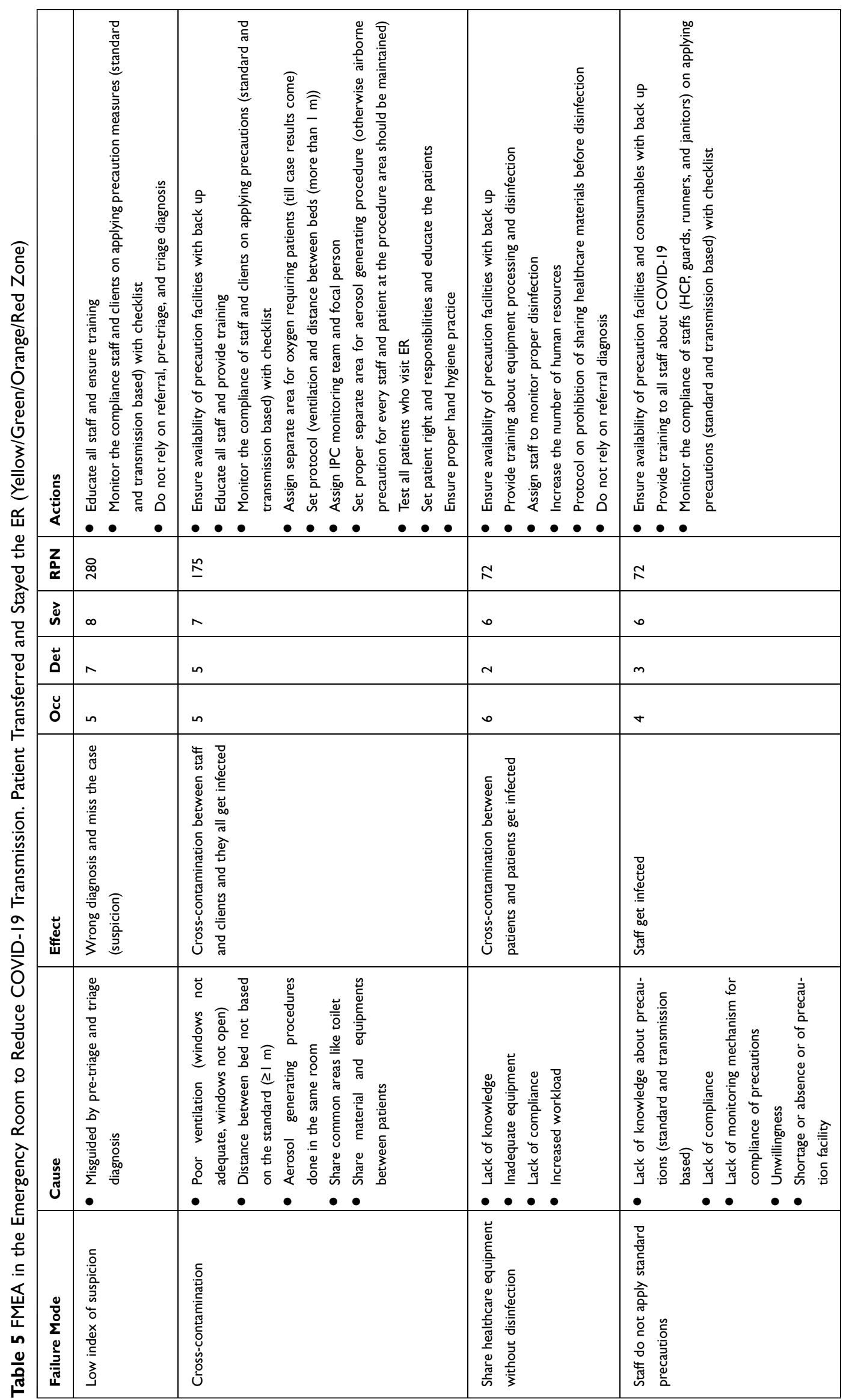




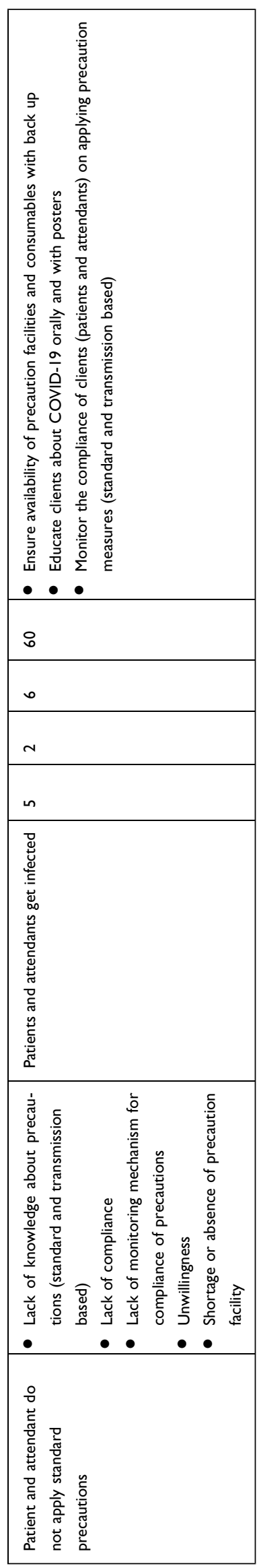

We believe that significant improvement can be brought by ensuring the availability of precaution facilities with back up, educating clients about COVID-19 orally and with posters, monitoring the compliance of clients on applying precaution measures with the checklist, by not allowing anyone on the gate to enter without a face mask and ensuring $24 * 7$ availability of screening at the gate and assign a staff member to be responsible. Measures listed under Table 1 were applied to curve the effect and strict monitoring of face mask use at the gate and increasing access for the hygienic facility were among actions implemented.

The most high-risk failure that could increase asymptomatic COVID-19 patient admission and transmission in the pre-triaging process is that Pre-triage does not detect the case (COVID-19) (RPN-192). This failure mode is difficult to detect and has a severe impact which has resulted from the asymptomatic presentation of the patient, the patient might give a false history, presence of atypical presentation, low index of suspicion, misguided by referral diagnosis, and lack of COVID-19 test for unsuspected cases because of resource limitations. Therefore, educating the public on the consequences of false patient history, and providing training for a high index of suspicion has a great role in this regard.

Having a low index of suspicion for COVID-19 as patients who were suspected to have COVID-19 were sent to an isolation ward in the pre-triage room makes a high-risk failure (RPN-336 and 280) in the triaging process and emergency room stay, respectively. A low index of suspicion is believed to have resulted from a misguidance from the diagnosis made in prior checkpoints in the pre-triage and triage area.

The main limitation of this study is its failure to study the impact of the interventions proposed. As part of the quality improvement tool, there needs to be continuous efforts to study the implementation of change ideas to generate possible mitigation strategies for future planning.

\section{Conclusion}

COVID-19 has unique characteristics that we have not encountered before, so proactive analysis of what will happen, what can cause the problems, its effect, and what has to be done to reduce the failures will make us stand ahead of the pandemic and result in reducing its devastating effect. Incorporating the FMEA tool as a quality improvement project during COVID-19 pandemic management is highly commendable and in the long run 
with disaster management at large as it has a great impact on identifying possible failures in a new system proactively and suggests possible solutions ahead of time that could help in reducing secondary transmission to patients and staff.

\section{Disclosure}

The authors report no conflicts of interest for this work.

\section{References}

1. World Health Organization. Director-General's Opening Remarks at the Media Briefing on COVID-19-11 March 2020. Available from: https://www.who.int/dg/speeches/detail/who-director-general-s-openin gremarks-at-the-media-briefing-on-covid-19-3-march-2020.

Accessed May 11, 2020.

2. Ethiopia Federal Ministry of Health (MOH) Report on CoVID-19; March 13, 2020

3. Lupia T, Scabini S, Pinna SM, Di Perri G, De Rosa FG, Corcione S. 2019-novel coronavirus outbreak: A new challenge. J Glob Antimicrob Resist. 2020;21:22-27. doi:10.1016/j.jgar.2020.02.021

4. Yanes-Lane M, Winters N, Fregonese F, et al. Proportion of asymptomatic infection among COVID-19 positive persons and their transmission potential: a systematic review and meta-analysis. PLoS One. 2020;15(11):e0241536. doi:10.1371/journal.pone.0241536

5. Teheran AA, Camero G, de la Guardia RP, et al. Epidemiological characterization of asymptomatic carriers of COVID-19 in Colombia. medRxiv. 2020.

6. Institute for Healthcare Improvement. Optimizing patient flow: moving patients smoothly through acute care settings. Innovation Series; 2003:1.
7. Hughes RG. Tools and strategies for quality improvement and patient safety. In:Hughes RG,editor Patient Safety and Quality: An EvidenceBased Handbook for Nurses. Agency for Healthcare Research and Quality (US); 2008:2-13

8. Chuang PT. Combining service blueprint and FMEA for service design. Serv Ind J. 2007;27(2):91-104. doi:10.1080/ 02642060601122587

9. Sorrentino P. Use of failure mode and effects analysis to improve emergency department handoff processes. Clin Nurse Spec. 2016;30 (1):28-37. doi:10.1097/NUR.0000000000000169

10. Chanamool N, Naenna T. Fuzzy FMEA application to improve decision-making process in an emergency department. Appl Soft Comput. 2016;43:441-453. doi:10.1016/j.asoc.2016.01.007

11. Shahrami A, Rahmati F, Kariman H, et al. Utilization of failure mode and effects analysis (FMEA) method in increasing the revenue of emergency department; a prospective cohort study. Emergency. 2013;1(1):1.

12. Derosier J, Stalhandske E, Bagian JP, et al. Using health care failure mode and effect analysis: the VA National Center for Patient Safety's prospective risk analysis system. Jt Comm J Qual Improv. 2002;28:2. doi:10.1016/s1070-3241(02)28025-6

13. Ofek F, Magnezi R, Kurzweil Y, Gazit I, Berkovitch S, Tal O. Introducing a change in hospital policy using FMEA methodology as a tool to reduce patient hazards. Isr J Health Policy Res. 2016;5 (1):30. doi:10.1186/s13584-016-0090-7

14. St. Paul's Hospital Millennium Medical College. Liaison Office Report. Addis Ababa, Ethiopia; 2020.

15. Premala N, Stefan S, Sam C, Lola EK. Failure modes and effect analysis to develop transfer protocols in the management of COVID19 patients. Br J Anaesth. 2020.

16. Levy N, Zucco L, Ehrlichman RJ, et al. Development of rapid response capabilities in a large COVID-19 alternate care site using failure modes and effect analysis with in situ simulation. Anesthesiology. 2020;133 (5):985-996. doi:10.1097/ALN.0000000000003521
Risk Management and Healthcare Policy

\section{Publish your work in this journal}

Risk Management and Healthcare Policy is an international, peerreviewed, open access journal focusing on all aspects of public health, policy, and preventative measures to promote good health and improve morbidity and mortality in the population. The journal welcomes submitted papers covering original research, basic science, clinical \& epidemiological studies, reviews and evaluations, guidelines, expert opinion and commentary, case reports and extended reports. The manuscript management system is completely online and includes a very quick and fair peer-review system, which is all easy to use. Visit http://www.dovepress.com/testimonials.php to read real quotes from published authors. 